\title{
Robust Real-Time Segmentation of Images and Videos Using a Smooth-Spline Snake-based algorithm
}

\author{
Frederic Precioso, Michel Barlaud, Thierry Blu, Member, IEEE, and Michael Unser, Fellow, IEEE
}

\begin{abstract}
This paper deals with fast image and video segmentation using active contours. Region-based active contours using level sets are powerful techniques for video segmentation, but they suffer from large computational cost. A parametric active contour method based on B-Spline interpolation has been proposed in [26] to highly reduce the computational cost, but this method is sensitive to noise. Here, we choose to relax the rigid interpolation constraint in order to robustify our method in the presence of noise: by using smoothing splines, we trade a tunable amount of interpolation error for a smoother spline curve. We show by experiments on natural sequences that this new flexibility yields segmentation results of higher quality at no additional computational cost. Hence, real-time processing for moving objects segmentation is preserved.
\end{abstract}

\section{INTRODUCTION}

W E address the problem of image and video segmentation using region-based active contours. The goal is to extract image regions corresponding to semantic objects. Image and video segmentation can be cast in a minimization framework by choosing a criterion which includes region and boundary functionals. Boundary functionals were first proposed by Kass $e t a l$. [21] and geodesic active contours by Caselles et al. [3], [4] for active contour segmentation. Region-based active contours were first introduced by Ronfard et al. [30] and Cohen et al. [10]. Chakraborty et al. [5] combined both boundary and region information for medical images segmentation. Then, Chesnaud et al. [9], Chan et al. [6], Zhu et al. [35], Paragios et al. [24], and Debreuve et al. [12] introduce region-based statistic descriptors for image or video segmentation. Jehan-Besson et al. [17], [20] address the segmentation problem where features of the region to be segmented are embedded in region functionals. In this framework, Gastaud et al. [13] propose a new approach introducing shape prior. This method uses a variational approach as opposed to previous work on shape prior, based on probabilistic methods [11]. The shape prior allows free form deformation [13] and is not restricted to a parametric deformation as in [8].

All these contour or region-based methods use a level-set approach which is accurate but time consuming. In this paper, we

Manuscript received October 6, 2003; revised May 10, 2004. The associate editor coordinating the review of this manuscript and approving it for publication was Dr. Nicolas Merlet.

F. Precioso and M. Barlaud are with the Laboratoire I3S-UPRES-A 6070 CNRS, Université de Nice, F-06903 Sophia-Antipolis, France (e-mail: frederic.precioso@i3s.unice.fr; michel.barlaud@i3s.unice.fr).

T. Blu and M. Unser are with the Biomedical Imaging Group, STI/IOA, Swiss Federal Institute of Technology, CH-1015 Lausanne, Switzerland (e-mail: thierry.blu@epfl.ch; michael.unser@epfl.ch).

Digital Object Identifier 10.1109/TIP.2005.849307 propose a parametric active contour evolution based on a cubic spline contour [2].

In Section II, we present a survey of the region-based criterion, the derivation of the criterion and computation of the velocity vector.

In Section III, we propose a cubic B-spline implementation. Cubic B-splines preserve $C^{2}$ regularity and have excellent approximation properties [32] which means that, for a given accuracy, fewer samples are needed than with other parametric methods; moreover, fast algorithms are available for B-splines, which greatly reduces the computation cost.

Unfortunately, interpolation methods are not robust to noise. This is why we propose to use smoothing splines [33] in the $\mathrm{B}$-spline interpolation approach of [27]. These curves preserve the implementation advantages as the B-splines while softening the interpolation constraint. The relaxation of the interpolation condition is traded for an optimal increase of the smoothness of the spline snake. A smoothness parameter controls the amount of relaxation.

In Section IV, we compare the influence of the smoothing spline parameter with the curve-length regularization coefficient. Finally, we show some experiments on real video sequences.

\section{REgion-BASED Active Contours}

\section{A. Criterion and Velocity}

Let us define a general segmentation criterion. For each frame of the sequence, we search a background region $\Omega_{\text {out }}$, and object regions $\Omega_{\text {in }}$ with a common boundary $\Gamma$ (Fig. 1). Thus, the criterion includes both region and boundary functionals

$$
\begin{aligned}
& J\left(\Omega_{\text {out }}, \Omega_{\text {in }}, \Gamma\right)=\underbrace{\int_{\Omega_{\text {out }}} k_{\text {out }}\left(\Omega_{\text {out }}\right) d \sigma+\int_{\Omega_{\text {in }}} k_{\text {in }}\left(\Omega_{\text {in }}\right) d \sigma}_{\text {Region terms }} \\
& +\underbrace{\int_{\Gamma} \beta d s}_{\text {Boundary term }} .
\end{aligned}
$$

In this criterion, $k_{\text {out }}$ is the descriptor of the unknown background domain $\Omega_{\text {out }}, k_{\text {in }}$ is the descriptor of the unknown object domain $\Omega_{\mathrm{in}}$ and $\beta$ is the weight of the regularization.

Since we use statistical descriptors (for $k_{\text {in }}$ and $k_{\text {out }}$ ), such as mean, variance, or region histograms, the entropy descriptors are globally attached to the region $\Omega$ [9]. In the variational 


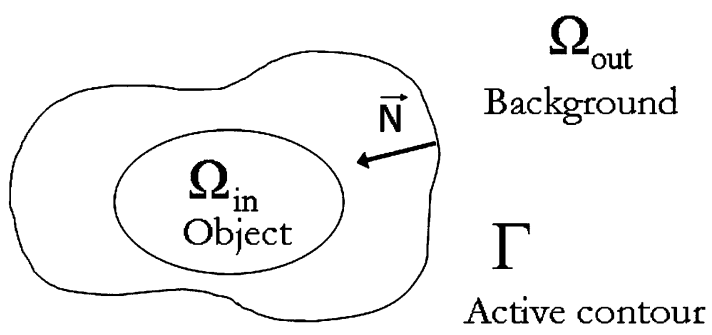

Fig. 1. Domain definition.

Eulerian method proposed by Jehan-Besson et al. [20], for a region-based active contour segmentation, the authors introduce a dynamical scheme (shape gradient method) in the criterion. Hence, regions become continuously dependent on an evolution parameter $\tau$.

The criterion $J\left(\Omega_{\text {out }}(\tau), \Omega_{\text {in }}(\tau), \Gamma(\tau)\right)$ is denoted by $J(\tau)$. Thus, the computation of the derivative provides

$$
\begin{aligned}
J^{\prime}(\tau)= & \underbrace{\int_{\Omega_{\mathrm{out}}(\tau)} \frac{\partial k_{\mathrm{out}}}{\partial \tau} d \sigma+\int_{\Omega_{\mathrm{in}}(\tau)} \frac{\partial k_{\mathrm{in}}}{\partial \tau} d \sigma}_{(a)} \\
& +\underbrace{\int_{\Gamma(\tau)}\left(k_{\mathrm{out}}-k_{\mathrm{in}}\right)(\boldsymbol{v} \cdot \boldsymbol{N}) d s}_{(b)} \\
& +\underbrace{\int_{\Gamma(\tau)}(-\beta \cdot \kappa+\nabla \beta \cdot \boldsymbol{N})(\boldsymbol{v} \cdot \boldsymbol{N}) d s}_{(c)}
\end{aligned}
$$

where $\kappa$ is the curvature of the contour, $v$ is the velocity of $\Gamma(\tau)$, and $N$ is the unit inward normal to $\Gamma(\tau)$.

The terms $(a)$ are deduced from the variation of the descriptors with the region. The term $(b)$ is deduced from the variation of the region, and the classical term $(c)$ comes from the derivation of the Boundary term in (1) [4]. Complete proofs are available in [17], [19], and [20].

The active contour $\Gamma(\tau)$ evolves from an initial position $\Gamma(0)$ toward the object with a velocity $v$ in the direction of $N$, the inward normal vector of the active contour

$$
\left\{\begin{array}{l}
\frac{\partial \Gamma(\tau)}{\partial \tau}=v \boldsymbol{N} \\
\Gamma(0)=\Gamma_{0}
\end{array}\right.
$$

The velocity expression is deduced from the derivative (2)

$$
v=\mathbf{A}+k_{\mathrm{in}}-k_{\mathrm{out}}+\beta \kappa .
$$

- A represents local terms which are computed from the two first terms $(a)$ in (2). In Section IV, we will detail the expression of $\mathbf{A}$ and then the velocity $v$ for two applications: segmentation of homogenous regions and segmentation of moving objects.

- $\beta$ is a constant.

\section{B. Implementation}

Region-based active contour evolution can be implemented in two different ways.
- Implicitly, based on the level-set approach [19], [20], [23]. Such a method provides an implicit management of topological changes and yields accurate results, but it suffers from a high computational cost.

- Explicitly, using active parametric contours. Such a method reduces the computational cost substantially and provides a complete control of the data size. The accuracy of the results is dependent on the noise level of the sequence. Using smoothing splines is likely to introduce robustness in this method.

\section{TOWARDS CUBIC SMOOTHING SPLINES}

\section{A. Cubic Spline Interpolation}

The evolution velocity is now computed only at sampling points along a spline active contour. Cubic spline curves are parametric curves $S(t)=(x(t), y(t))$ where $x(t)$ and $y(t)$ are cubic polynomials on each segment $t_{k} \leq t \leq t_{k+1}$ and are smoothly (twice continuously differentiable) connected between segments. Here, we assume that there are $n$ such segments parametered by $t_{0}, t_{1}, \ldots t_{n-1}$ with the assumption $t_{n}=t_{0}$ and that we are given the $n$ sampling points $P_{k}=S\left(t_{k}\right)$.

Each segment $t_{k} \leq t \leq t_{k+1}$ is expressed as a cubic polynomial [1]

$$
\begin{aligned}
S(t)=Q_{k-1} B_{k-3}^{3}(t)+ & Q_{k} B_{k-2}^{3}(t) \\
& +Q_{k+1} B_{k-1}^{3}(t)+Q_{k+2} B_{k}^{3}(t) .
\end{aligned}
$$

$B_{k}^{3}(t)$ is a nonuniform B-spline function; the $n$ parameters of the model are the B-spline coefficients $Q_{k}$ called control points. These coefficients can be specified by solving for $Q_{k}$ the $n$ equations $S\left(t_{k}\right)=P_{k}$.

The B-spline function $B_{k}^{3}(t)$ is a piecewise cubic polynomial that depends on the $n$ values $t_{k}$ of the curve parameter at the sampling points.

Irregular sampling of $t$ is intuitively more pertinent, as regards active contour propagation, than uniform sampling. This is the option chosen by, e.g., Pottmann et al. [25] and Yang et al. [34], who propose to optimize the parameterization of the spline curve for approximating a target curve, as well as in other approaches based on arbitrary parameterizations (chord length, centripetal, Foley, etc.). However, building the nonuniform spline curve requires the computation of $n$ different polynomials $B_{k}^{3}$ which is time consuming. To overcome this problem, we have proposed a regular sampling approach [27], i.e., $t_{k}=k$, to represent the active contour using uniform B-spline functions. In that case, after reparameterizing the curve, the B-spline function $B_{k}^{3}(t)$ is independent of the segment considered on the curve. We can write $B_{k}^{3}(t)=\beta^{3}(t-k)$ where the centered B-spline of degree $3 \beta^{3}(t)$ is a bell-shaped, symmetrical function, as shown in Fig. 2, defined by

$$
\beta^{3}(t)= \begin{cases}\frac{2}{3}-|t|^{2}+\frac{|t|^{3}}{2}, & 0 \leq|t|<1 \\ \frac{(2-|t|)^{3}}{6}, & 1 \leq|t|<2 \\ 0, & 2 \leq|t|\end{cases}
$$




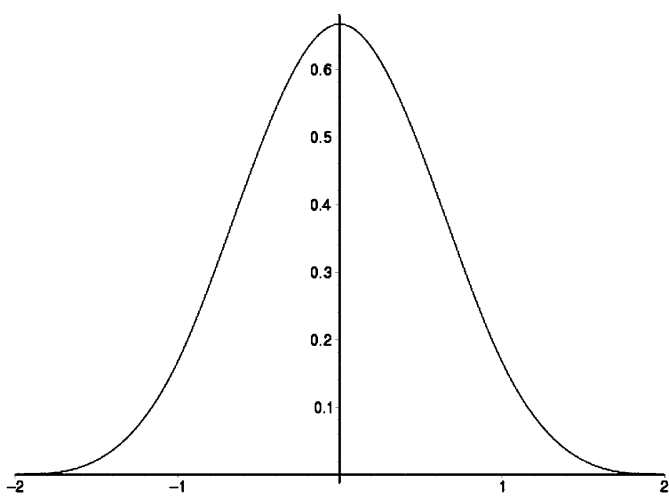

Fig. 2. Centered B-spline of degree 3.

The arc (5) with (6) becomes [1]

$$
\begin{aligned}
S(t)= & \left(-\frac{1}{6} Q_{k-1}+\frac{1}{2} Q_{k}-\frac{1}{2} Q_{k+1}+\frac{1}{6} Q_{k+2}\right) t^{3} \\
& +\left(\frac{1}{2} Q_{k-1}-Q_{k}+\frac{1}{2} Q_{k+1}\right) t^{2} \\
& +\left(-\frac{1}{2} Q_{k-1}+\frac{1}{2} Q_{k+1}\right) t \\
& +\frac{1}{6} Q_{k-1}+\frac{2}{3} Q_{k}+\frac{1}{6} Q_{k+1} \text { for } t \in[k, k+1] .
\end{aligned}
$$

The computation cost of regular sampling is lower than handling a specific equation for each segment $t_{k} \leq t \leq t_{k+1}$ of a nonuniform B-Spline curve.

Moreover, the control points $Q_{k}$ can be obtained from the sampling points $P_{k}$ using a fast filtering algorithm when the curve parameter is sampled uniformly (see Appendix I).

Indeed, each interpolated point $P_{k}$ corresponds to the polynomial expression value $S(t)$ when $t=k$. From (7), we obtain the relation between sampling points $P_{k}$ and control points (B-spline coefficients) $Q_{k}$

$$
S(k)=P_{k}=\frac{1}{6}\left(Q_{k-1}+4 Q_{k}+Q_{k+1}\right) .
$$

This relation can be written as a convolution

$$
Q_{k}=\left(B_{1}^{3}\right)^{-1} * P_{k}
$$

where $B_{1}^{3}$ is the discrete cubic B-spline kernel.

The inverse convolution operator is defined by

$$
B_{1}^{3}(z)^{-1}=\frac{6}{z+4+z^{-1}}
$$

Using the prefiltering approach exposed in [33], the inverse convolution operator $\left(B_{1}^{3}\right)^{-1}$ is computed efficiently from a cascade of first order causal and anti-causal recursive filters (see details in Appendix I). The control points $Q_{k}$ are computed from sampling points $P_{k}$ using this fast filtering algorithm.

Cubic splines provide good interpolation accuracy at low computational cost [32]. Moreover, these curves have several interesting properties: They are twice continuously differentiable, which allows to build a $C^{2}$-regular curve. Thus, the normal vector and the curvature, involved in the velocity equation, can be computed exactly at every sampling point. In addition, such curves minimize the following criterion:

$$
\int_{\Gamma}\left\|\mathcal{C}^{\prime \prime}(t)\right\|^{2} d t
$$

under interpolatory constraints [31]. Here, $\mathcal{C}(t)=(x(t), y(t))$ is a parametric description of the curve $\Gamma$ and $\mathcal{C}^{\prime \prime}(t)$ the second derivative of $\mathcal{C}(t)$ w.r.t. $t$; this functional is actually very close to the (squared) curvature $\kappa^{2}$ when the parameter $t$ is close to the curvilinear abscissa, as shown in [16].

Although we have obtained real-time accurate results with an implementation based on these B-spline curves [26], interpolation is not robust enough in the presence of noise. Thus, we propose to use a less constrained approximation method; namely, the smoothing spline method [28].

\section{B. Cubic Spline Approximation}

A smoothing spline is an approximation curve controlled by a parameter trading interpolation error for smoothness [29]. It minimizes the following criterion:

$$
\int_{\Gamma}\left\|\mathcal{C}^{\prime \prime}(t)\right\|^{2} d t+\frac{1}{\lambda} \sum\left(P_{k}-\mathcal{C}_{k}\right)^{2}
$$

where the $P_{k}$ s are the measured data points and $\mathcal{C}_{k} \mathrm{~s}$ are points, on the curve, joining of polynomial pieces.

The result is still a cubic spline, but it does not satisfy anymore the interpolation condition exactly. The interpolation error has been converted into increased smoothness-smaller energy of the second derivative.

The first term of (12) can be developed as

$$
\begin{aligned}
\left\|\mathcal{C}^{\prime \prime}\right\|^{2} & =\frac{\left|x^{\prime} x^{\prime \prime}+y^{\prime} y^{\prime \prime}\right|^{2}}{x^{\prime 2}+y^{\prime 2}}+\frac{\left|x^{\prime} y^{\prime \prime}-x^{\prime \prime} y^{\prime}\right|^{2}}{x^{\prime 2}+y^{\prime 2}} \\
& =\left|\frac{d}{d t} \sqrt{x^{\prime 2}+y^{\prime 2}}\right|^{2}+\left(x^{2}+y^{\prime 2}\right)\left|\frac{d}{d t} \arctan \left(\frac{y^{\prime}}{x^{\prime}}\right)\right|^{2} \\
& =s^{\prime \prime 2}+s^{\prime 2} \phi^{\prime 2}
\end{aligned}
$$

where $s$ is the curvilinear abscissa of the curve $\Gamma$, and $\phi(t)$ is the angle of the tangent to the curve at $\mathcal{C}(t)$. This shows that the smoothing part in (12) can be rewritten as a sum of two positive terms

$$
\int_{\Gamma}\left\|\mathcal{C}^{\prime \prime}(t)\right\|^{2} d t=\int_{\Gamma} s^{\prime \prime 2}(t) d t+\int_{\Gamma} s^{2}(t) \phi^{\prime 2}(t) d t .
$$

Thus, the decrease of $\int_{\Gamma}\left\|\mathcal{C}^{\prime \prime}(t)\right\|^{2} d t$ facilitates the decrease of $\int_{\Gamma} s^{\prime \prime 2}(t) d t$ and $\int_{\Gamma} s^{\prime 2}(t) \phi^{\prime 2}(t) d t$ :

- the first term of (13) represents the average variation of curvilinear abscissa. Thus, decreasing this term tends to improve the curve sampling uniformity;

- when the sampling is nearly uniform-i.e., $s^{\prime}(t) \approx$ Constant, which is favored by the decrease of the first term - the second term of (13) is lower bounded by the square of (using Cauchy-Schwartz inequality)

$$
\frac{\operatorname{length}(\Gamma)}{(\# \text { control points })^{3 / 2}} \int_{\Gamma}\left|\phi^{\prime}(t)\right| d t
$$

which is the average tangent angle variation over the curve. Preventing this quantity from being large also 


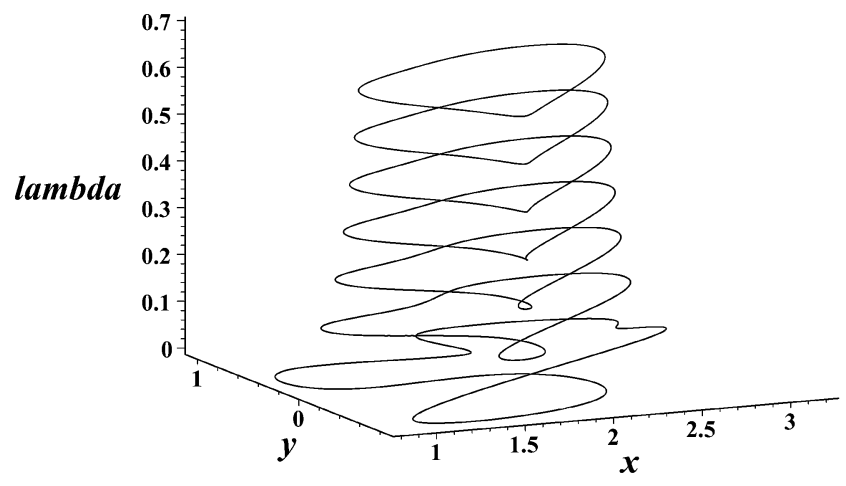

Fig. 3. Evolution from cubic spline interpolation $(\lambda=0)$ toward cubic smoothing spline approximation $(\lambda=0.7)$.

prevents loops in the curves. This is because each loop increases the value of $\int_{\Gamma}\left|\phi^{\prime}(t)\right| d t$ by $\pi$ at least.

Fig. 3 shows the evolution of a cubic spline curve, based on 10 data points, from regular interpolation (for $\lambda=0$ ) toward a smoothing spline approximation (for $\lambda=0.7$ ). This figure shows that loops are avoided as the smoothness parameter $\lambda$ increases.

In Figs. 4 and 5, we have plotted the variance of the curvilinear abscissa $\int_{\Gamma} s^{\prime \prime}(t)^{2} d t$ and the interpolation error of smoothing splines as functions of the smoothness parameter $\lambda$.

The curve in Fig. 4 confirms that the interpolation error increases slightly with $\lambda$. Indeed, the amount of missclassified pixels, between the smoothing spline segmentation and a segmentation of reference, increases with $\lambda$, but only up to $1.6 \%$ of the size of the object.

Fig. 5 shows that, on the contrary, the curvilinear abscissa variance decreases with $\lambda$.

The relation between sampling points $P_{k}$ and control points $Q_{k}$ (B-spline coefficients) can be written as a convolution

$$
Q_{k}=\left(S_{\lambda}^{3}\right)^{-1} * P_{k}
$$

with

$$
S_{\lambda}^{3}(z)^{-1}=\frac{6}{z+4+z^{-1}+6 \lambda\left(z^{-2}-4 z^{-1}+6-4 z+z^{2}\right)} .
$$

This transfer function is factorized into a product of causal and anticausal responses

$$
\frac{b}{\lambda} \cdot \frac{1}{1+a z^{-1}+b z^{-2}} \cdot \frac{1}{1+a z+b z^{2}}
$$

where $a=-\left(z_{0}+z_{1}\right)$ and $b=z_{0} z_{1}$ are real quantities obtained from the two poles $z_{0}$ and $z_{1}$ of $S_{\lambda}^{3}(z)^{-1}$ that are inside the unit circle. This prefiltering approach provides an efficient method to compute the smoothing spline coefficients (see details in Appendix II).

The positive parameter $\lambda$ quantifies the tradeoff between interpolation error and regularity. For $\lambda=0$, no interpolation error is allowed, and, thus, we get interpolating splines. When $\lambda$ increases, a larger amount of interpolation error is allowed; hence, the spline snake is smoother and its sampling more regular. We show in Section IV the benefits of this new approach for the segmentation of noisy images.

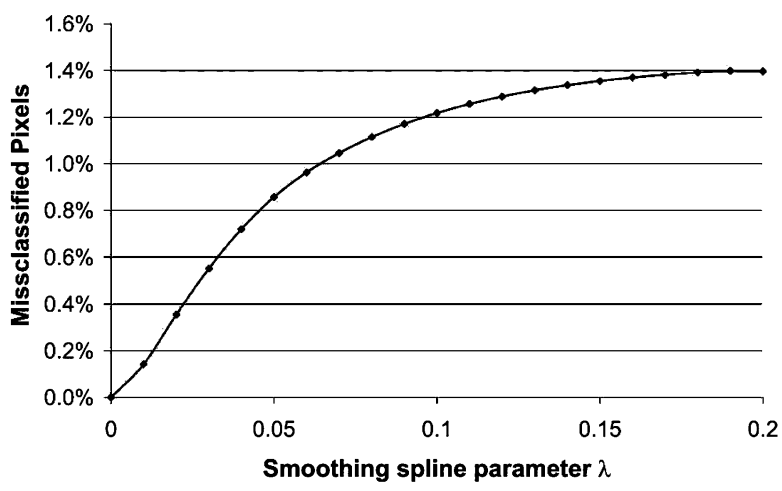

Fig. 4. Accuracy decreases slightly with $\lambda$.

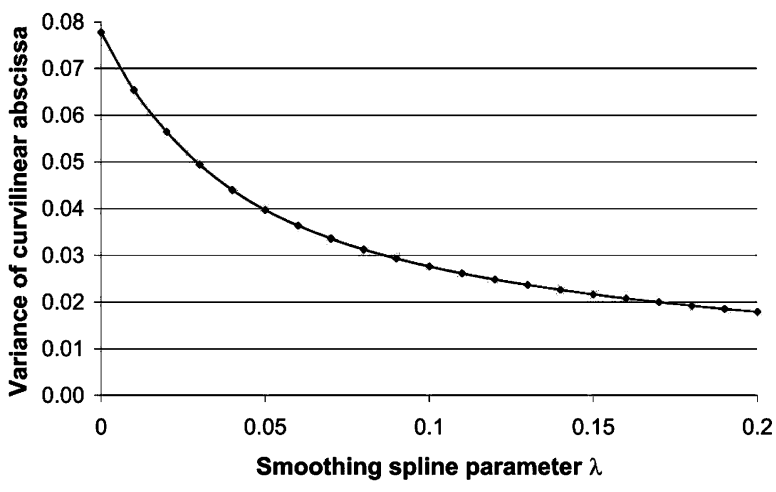

Fig. 5. Sampling uniformity increases with $\lambda$.

\section{Segmentation of Noisy Data}

In this section, we present results of segmentation of static images and segmentation of video sequences. We compare the respective influence of the smoothing spline parameter $\lambda$ and the contour length regularization parameter $\beta$ on the segmentation quality. We first show results of a segmentation based on a homogeneity criterion.

\section{A. Segmentation of Homogeneous Regions}

1) Grayscale Still Images: In this example, the images are osteoporosis medical images. The goal is to segment bone regions in the image. We consider the functional (1) where $k_{\text {out }}$ and $k_{\text {in }}$ are descriptors of the bone homogeneity. The region homogeneity is characterized by a function of the variance of luminance intensity. Let $\sigma_{\text {out }}^{2}$ and $\mu_{\text {out }}$ represent respectively the variance and mean of $\Omega_{\text {out }}(\tau), \sigma_{\mathrm{in}}^{2}$, and $\mu_{\mathrm{in}}$ represent, respectively, the variance and mean of $\Omega_{\mathrm{in}}(\tau)$, and $\Phi(r)$ a positive $C^{1}(\mathbb{R})$ function, for instance, $\Phi(r)=\log \left(1+r^{2}\right)$.

Thus, the criterion to be minimized is

$$
\begin{aligned}
J\left(\Omega_{\text {out }}, \Omega_{\text {in }}, \Gamma\right)=\int_{\Omega_{\text {out }}} \Phi & \left(\sigma_{\text {out }}^{2}\right) d \sigma \\
& +\int_{\Omega_{\text {in }}} \Phi\left(\sigma_{\text {in }}^{2}\right) d \sigma+\int_{\Gamma} \beta d s
\end{aligned}
$$

where $\beta$ is a positive constant. 

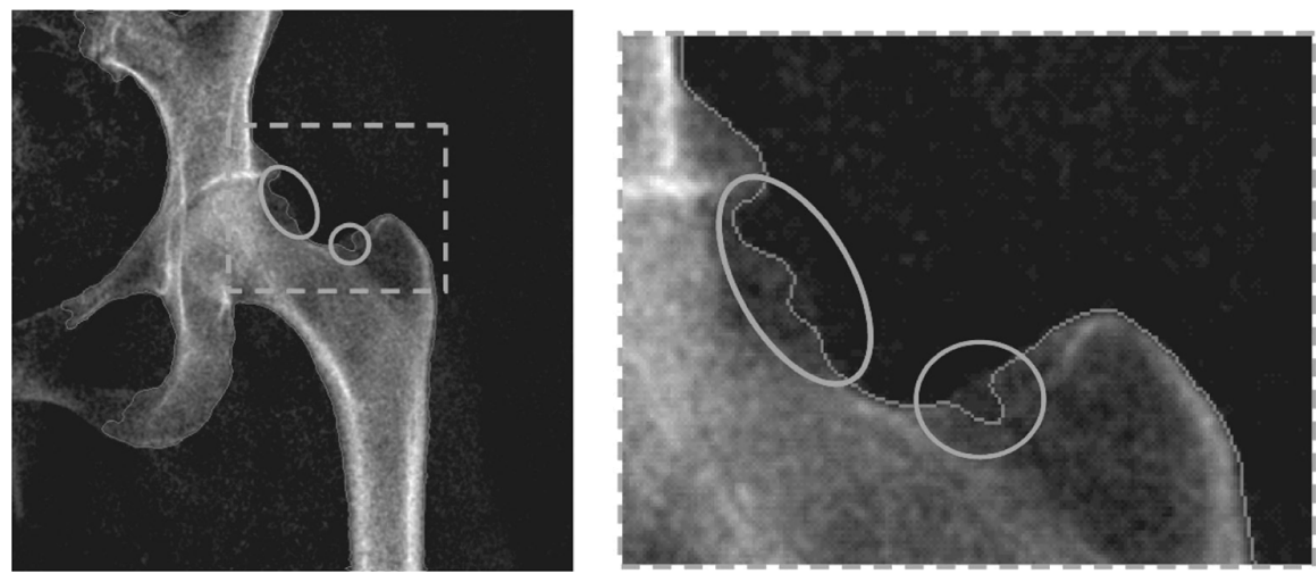

Fig. 6. Regular spline segmentation with $\lambda=0$ and length penalty $\beta=10$.
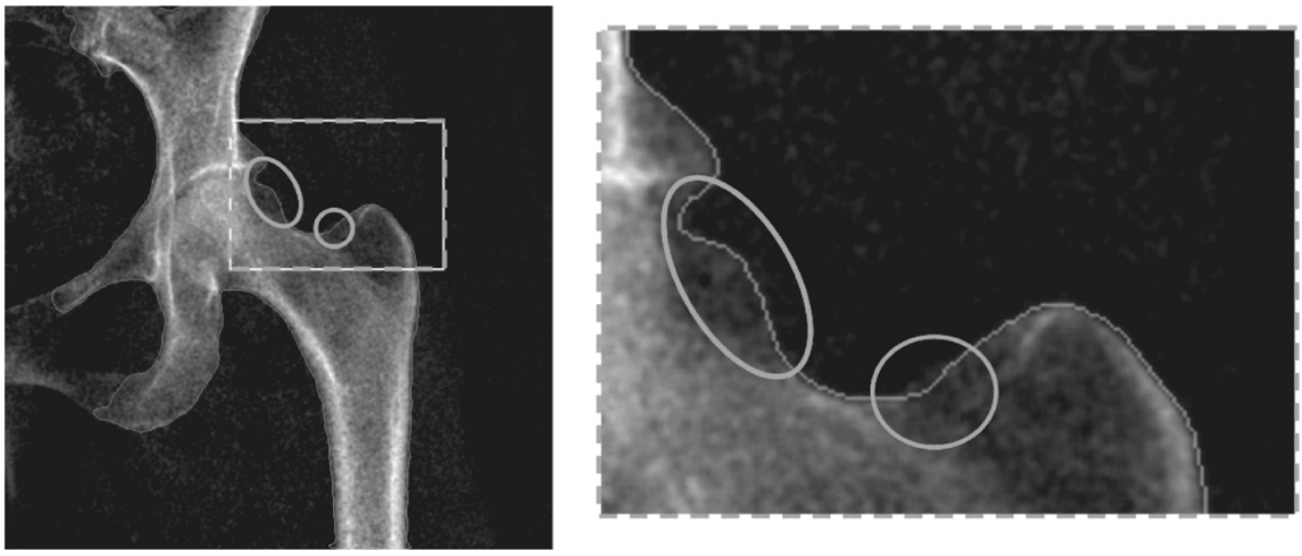

Fig. 7. Smoothing spline segmentation with $\lambda=0.08$ and $\beta=0$.

Using (2), the derivative of the functional $J$ with respect to $\tau$ is

$$
\begin{aligned}
J^{\prime}(\tau)=-\int_{\Gamma(\tau)}\left[\begin{array}{l}
\Phi \\
\end{array}\right. & \left(\sigma_{\text {in }}^{2}\right)-\Phi\left(\sigma_{\text {out }}^{2}\right)+\beta \kappa \\
& +\Phi^{\prime}\left(\sigma_{\text {in }}^{2}\right)\left[\left(I-\mu_{\text {in }}\right)^{2}-\sigma_{\text {in }}^{2}\right] \\
& \left.-\Phi^{\prime}\left(\sigma_{\text {out }}^{2}\right)\left[\left(I-\mu_{\text {out }}\right)^{2}-\sigma_{\text {out }}^{2}\right]\right] \\
& \cdot(\boldsymbol{v} \cdot \boldsymbol{N}) d s
\end{aligned}
$$

where $\kappa$ is the curvature of the contour and $\beta$ is a constant.

More details and proofs are available in Jehan-besson et al. [19]. In order to find a local extremum of the criterion (17), as the authors proposed, we evolve a curve using the steepest descent method. Thus, we obtain the following evolution equation:

$$
\frac{\partial \Gamma(\tau)}{\partial \tau}=\boldsymbol{v} \cdot \boldsymbol{N}
$$

Thus, the expression (4) of the velocity $v$ is known

$$
\begin{aligned}
v= & \Phi\left(\sigma_{\text {in }}^{2}\right)-\Phi\left(\sigma_{\text {out }}^{2}\right)+\beta \kappa \\
& +\Phi^{\prime}\left(\sigma_{\text {in }}^{2}\right)\left[\left(I-\mu_{\text {in }}\right)^{2}-\sigma_{\text {in }}^{2}\right] \\
& -\Phi^{\prime}\left(\sigma_{\text {out }}^{2}\right)\left[\left(I-\mu_{\text {out }}\right)^{2}-\sigma_{\text {out }}^{2}\right] .
\end{aligned}
$$

This velocity makes the B-spline active contour evolve toward the minimum of the energy criterion (17). Thus, the competition between the region inside the contour $\Omega_{\text {in }}(\tau)$ and the region outside $\Omega_{\text {out }}(\tau)$ leads to increase the homogeneity of both regions. However, the images are corrupted by acquisition noise and by the noise of nonbone tissues (muscles, fat, etc.).

Fig. 6 shows the convergence using the cubic spline interpolation with length penalty method. The length penalty provides smoothness to the contour. However, the acquisition noise corrupts the segmentation quality in the area of interest for osteoporosis diagnostic. The smoothness of the contour depends only on the length penalty parameter $\beta$.

Fig. 7 shows the convergence using the new smoothing spline method. The flexibility of the smoothing splines provides an accurate bone segmentation without being corrupted by the noise. The smoothness of the contour depends only on the smoothing spline parameter $\lambda$.

Fig. 8 shows the robustness of the new smoothing spline method regarding $\lambda$ parameter variations. Between the left and the right picture on the top row, $\lambda$ is only increased from 0 to 0.01 [Fig. 8(a) and (b)], but still the accuracy of the segmentation is highly improved and the most efficient results are almost reached. If we increase again $\lambda$ ten times, up to 0.1 , [Fig. 8(c)], the segmentation is smooth but still very good. By increasing $\lambda$ ten times [Fig. 8(d)], the contour is too smooth but remains robust. Additional experiments with $\lambda=10$ and 100 indicate 


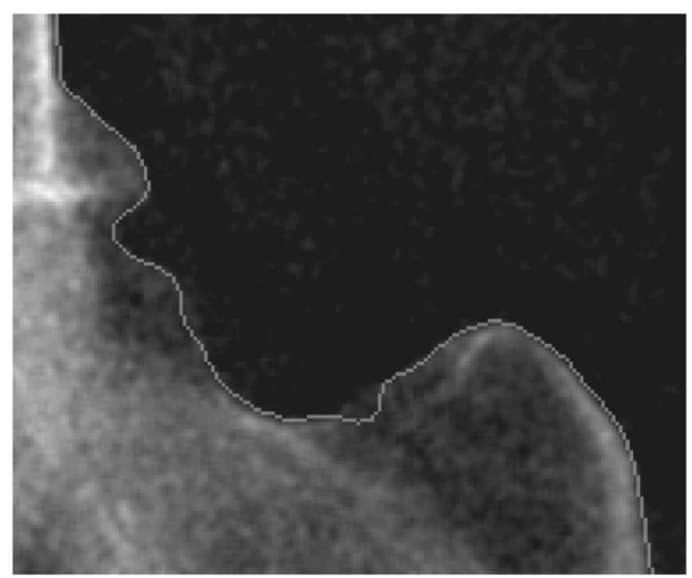

(a)

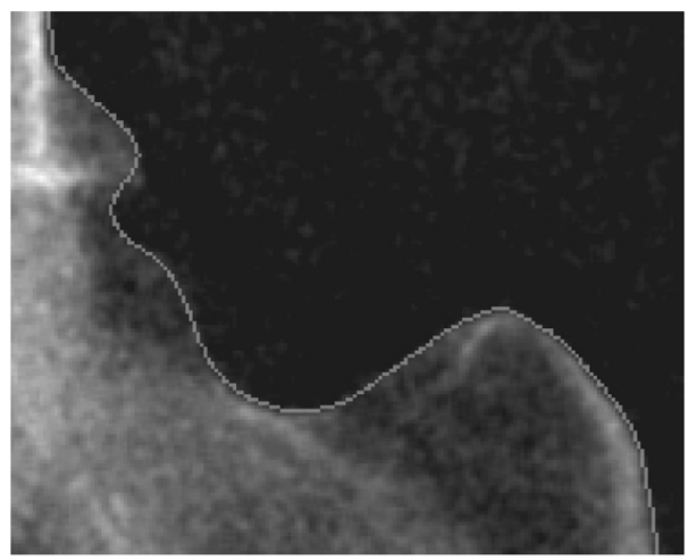

(c)

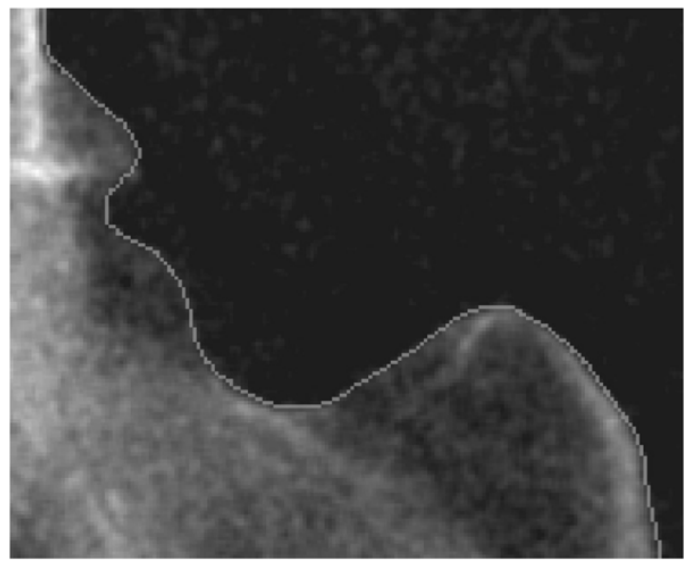

(b)

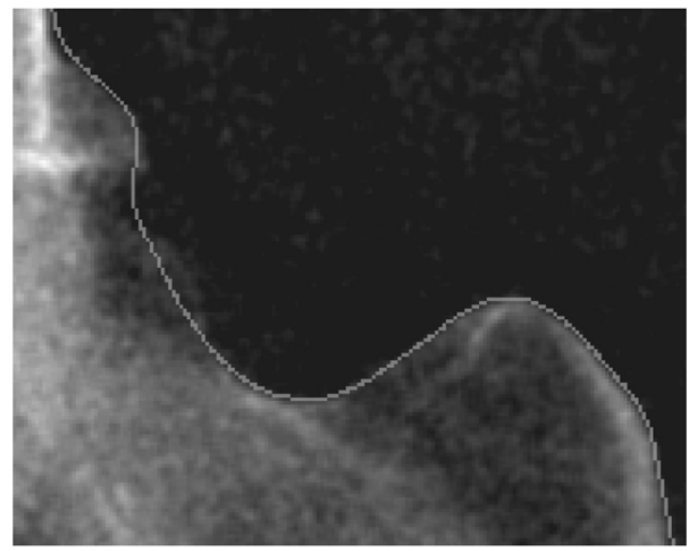

(d)

Fig. 8. Robustness regarding Smoothing spline parameter $\lambda$ variations. (a) Interpolation $\lambda=0$ and $\beta=0$. (b) Approximation $\lambda=0.01$ and $\beta=0$. (c) Approximation $\lambda=0.1$ and $\beta=0$. (d) Approximation $\lambda=1$ and $\beta=0$.

that even if the contour is too smooth to preserve a sufficient segmentation accuracy, the structure of the contour remains stable which is not true with variations of $\beta$, the weight on the length penalty. Such relative robustness of the snake with respect to $\lambda$ suggests that we can determine a range for standard values of this parameter. This first experiment provides a range of $[0.1,1]$.

The contour is sampled with 512 knots and the size of the image is $512 \times 512$. The segmentation is obtained in $25 \mathrm{~s}$ with a Pentium IV at $2.6 \mathrm{GHz}$. It has to be pointed out that most of this computation time is spent in evaluating the variance of the object domain and of the background. Indeed, segmentations with 256 and 128 knots, obtained in 24 s, confirm it: The cost of the approximation amounts to a few percents of the full computation.

To improve the computation time of our algorithm with such descriptors based on area moments (mean of the intensity, variance, etc.), a perspective would be to implement the method of Jacob et al. [15] for an exact computation of the area moments of spline curves.

2) Color Video Sequences: As detailed by Jehan et al. in [19], [20], the homogeneity in color images is related to the determinant of the covariance matrix for Gaussian distributions.
Yezzi et al. [22] and Herbulot et al. [14] extended this framework to the more general case of Entropy descriptors without Gaussian distribution hypothesis.

In these experiments, regions of interest are regions of homogeneous color, like the face on the sequence Erik. The color images are in the RGB color space. Let us define the joint probability distribution

$$
q\left(I_{R}(x), I_{G}(x), I_{B}(x), x \in \Omega\right)
$$

and the joint entropy, between the three channels of the image, on the domain $\Omega$

$$
\begin{aligned}
H_{\mathrm{RGB}}(\Omega)=- & \int_{\Omega} q\left(I_{R}(x), I_{G}(x), I_{B}(x), x \in \Omega\right) \\
& \times \ln q\left(I_{R}(x), I_{G}(x), I_{B}(x), x \in \Omega\right) d x .
\end{aligned}
$$

The segmentation of homogeneous regions of a color video sequence is achieved by region competition between the background $\Omega_{\text {out }}$ and the object $\Omega_{\mathrm{in}}$, minimizing the following criterion:

$$
J\left(\Omega_{\text {in }}, \Omega_{\text {out }}, \Gamma\right)=H_{\mathrm{RGB}}\left(\Omega_{\text {in }}\right)+H_{\mathrm{RGB}}\left(\Omega_{\mathrm{out}}\right)+\int_{\Gamma} \beta d s .
$$




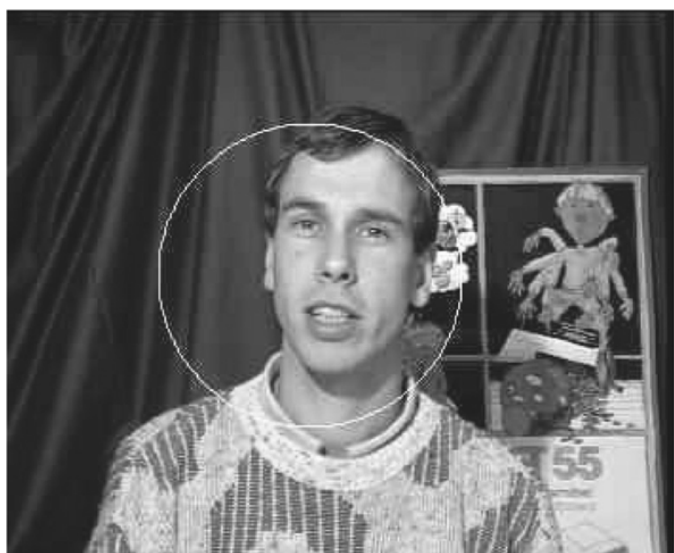

(a)

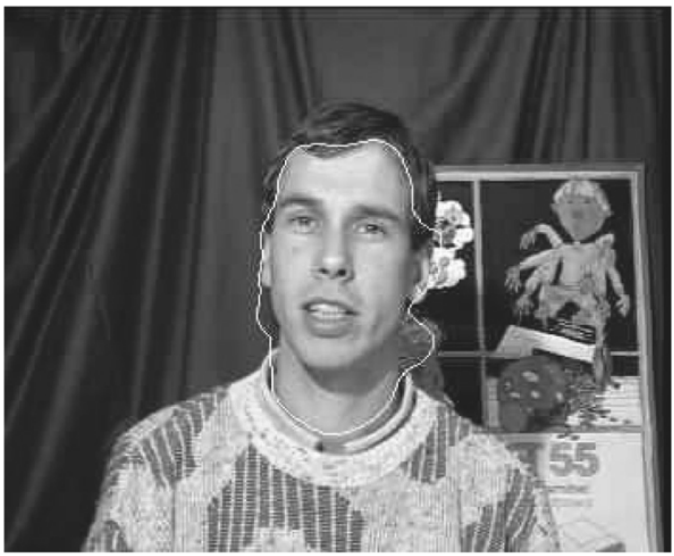

(c)

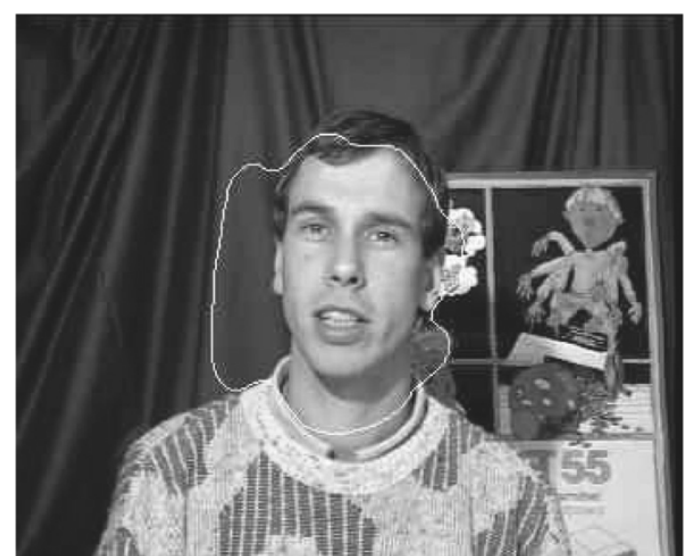

(b)

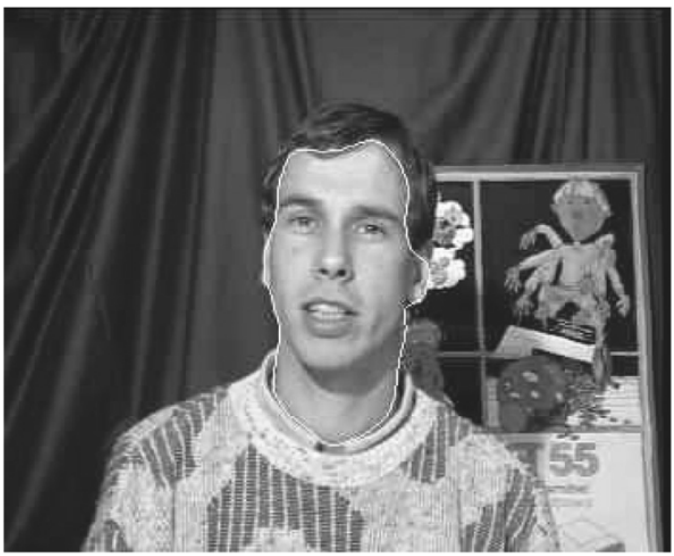

(d)

Fig. 9. Evolution of segmentation with the minimization of the criterion (21). (a) Initial curve. (b) Iteration 40. (c) Iteration 100. (d) Final segmentation (Iteration 430).

Thus, applying DREAMS method [20] to (21), the evolution equation based on the joint probability distributions is [14]

$$
\begin{aligned}
& \frac{\partial \Gamma}{\partial \tau}(\tilde{x})=\left[-q\left(I_{R}(\tilde{x}), I_{G}(\tilde{x}), I_{B}(\tilde{x}), \Omega\right)\right. \\
& \times\left(\ln q\left(I_{R}(\tilde{x}), I_{G}(\tilde{x}), I_{B}(\tilde{x}), \Omega\right)+1\right)-\frac{1}{|\Omega|} \\
& \times\left(H_{\mathrm{RGB}}(\Omega)-1+\int_{\Omega} K\left(I_{R}(x)-I_{R}(\tilde{x})\right.\right. \\
& \left.I_{G}(x)-I_{G}(\tilde{x}), I_{B}(x)-I_{B}(\tilde{x})\right) \\
& \left.\left.\times \ln q\left(I_{R}(x), I_{G}(x), I_{B}(x), \Omega\right) d x\right)\right] \mathbf{N}
\end{aligned}
$$

where

$$
K(x, y, z)=\frac{1}{2 \pi \sigma^{2}} \exp -\frac{x^{2}+y^{2}+z^{2}}{2 \sigma^{2}}
$$

is the Gaussian kernel involved in the Parsen window method.

Fig. 9 shows the evolution of the curve and Fig. 10 the segmentation of some frames of a sequence.

The data extracted from the histogram evolution are very sensitive to noise. This is why we use a smoothing B-spline approach which combines a very low computational cost and a global robustness to noisy data. The parameter $\lambda=0.1$ is in the standard range determined in the previous experiment, i.e., $[0.1,1]$.

\section{B. Moving Objects Segmentation}

Now, we present results obtained for the segmentation of moving objects in video sequences. This segmentation is based on motion detection. Our method is applied to the real "coastguard" video. The goal is to detect the boat of the coastguards in the sequence. We consider the functional (1) where $k_{\text {in }}$ and $k_{\text {out }}$ are, respectively, descriptor of moving objects and descriptor of the background. The descriptors have to take into account the camera motion in this sequence. Thus, the criterion to be minimized is

$$
\begin{aligned}
J\left(\Omega_{\text {out }}, \Omega_{\text {in }}, \Gamma\right)=\int_{\Omega_{\text {out }}} \mid I_{n}(\sigma)- & \operatorname{Proj}\left(I_{n-1}(\sigma)\right) \mid d \sigma \\
& +\int_{\Omega_{\text {in }}} \alpha d \sigma+\int_{\Gamma} \beta d s
\end{aligned}
$$

where $\operatorname{Proj}\left(I_{n-1}(\sigma)\right)$ is the projection of the image $I_{n-1}$ onto the referential of image $I_{n}$ in order to compensate for the motion of the camera. The camera motion model is based on a 6-parameter affine model. These parameters are computed with a robust 


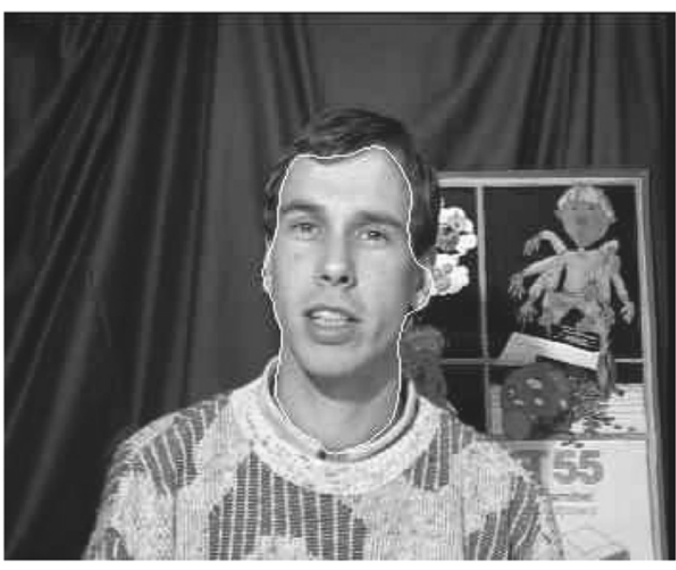

(a)

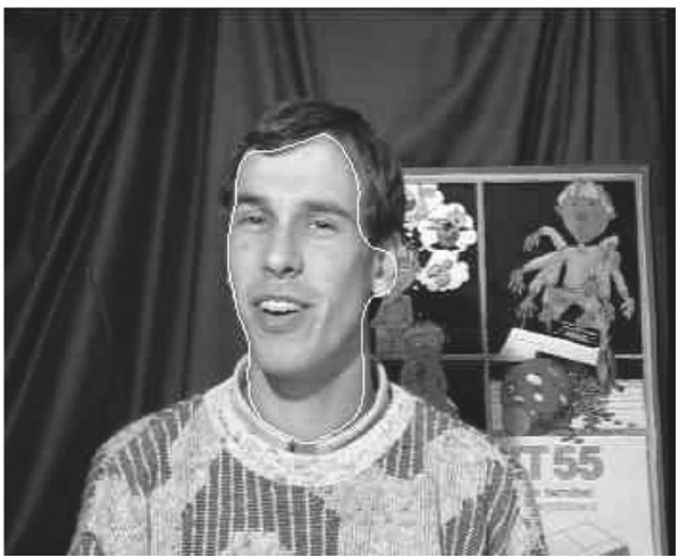

(c)

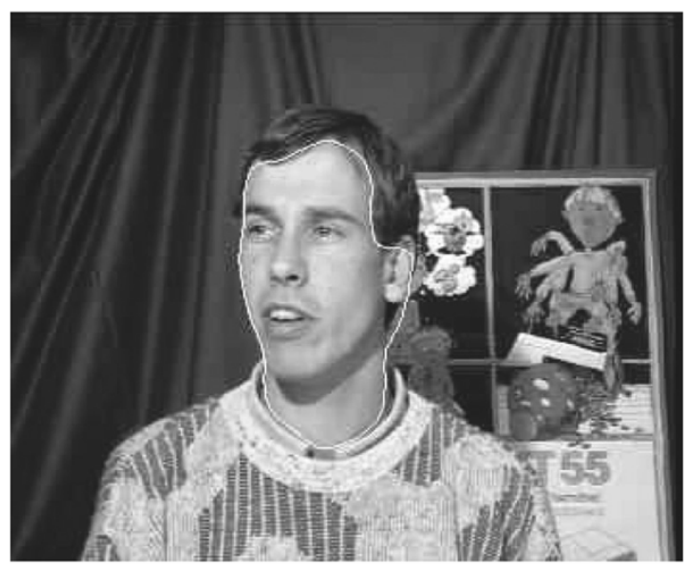

(b)

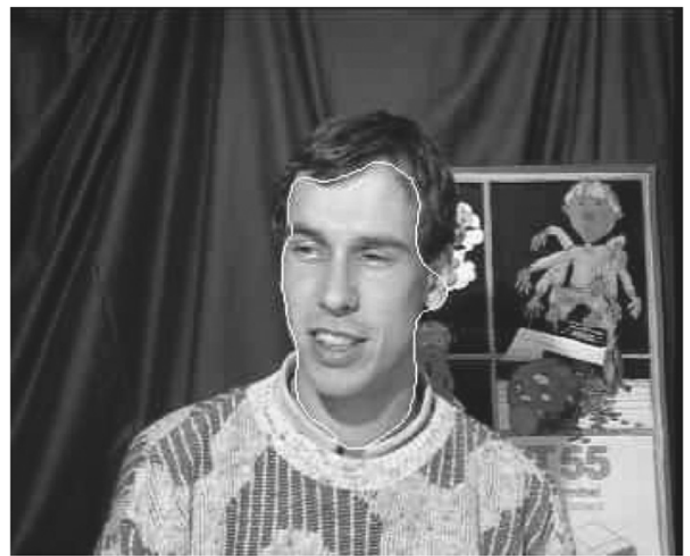

(d)

Fig. 10. Segmentation through a sequence. (a) Frame 10. (b) Frame 20. (c) Frame 30. (d) Frame 40.

estimation using motion vectors [7]. The motion field is evaluated by a classical block-matching algorithm between frames $I_{n}$ and $I_{n-1}$ [18]. $\beta$ and $\alpha$ are two positive constants.

The descriptors are region-independent. Thus, the local terms of the differentiation (2) disappear and the velocity (4) reduces to

$$
v=k_{\text {in }}-k_{\text {out }}+\beta \kappa \text {. }
$$

Since the descriptor $k_{\text {out }}=\left|I_{n}-\operatorname{Proj}\left(I_{n-1}\right)\right|$ is a temporal gradient, this local term is noise sensitive.

In the "coastguard" sequence Fig. 11(a), the wake of the boat behaves like noise for the background descriptor $k_{\text {out }}$. Thus, the contour evolution equation is corrupted by noise.

Fig. 11(b) shows the results using the cubic spline interpolation method [27]. The smoothness of the contour depends only on the contour length regularization parameter $\beta$. However, the foam in the wake of the boat is kept as part of the object.

Fig. 11(c) shows the results using the new smoothing spline method proposed here. The smoothness of the contour depends only on the smoothing spline parameter $\lambda$. Relaxing the rigid interpolation constraint brings an obvious improvement: The foam is not kept anymore, whereas the object is still reasonably well segmented.

This third experiment confirms the range of $[0.1,1]$, we determined, as standard values for the parameter $\lambda$.
Fig. 12 shows the computation time and the accuracy of the segmentation on the "coastguard" sequence, for the 5 first images. The contour is sampled with 64 knots and the size of the image is $352 \times 288$. The sequence is segmented with a Pentium IV at $2.6 \mathrm{GHz}$. The initial contour for the first frame is given by the image boundaries. For the frames 2, 3, 4, and 5, the initialization is provided by the final contour in the previous frame. The object in the first frame takes more time to be segmented because the initial contour is "far" from the object. For the other frames, the segmentation is achieved in less than $0.40 \mathrm{~s} /$ frame (with a Pentium IV at $2.6 \mathrm{GHz}$ ). Thus, the whole segmentation process should be ten times faster to provide results in real time. According to our knowledge, such a factor is not out of reach by optimization for an industry expert.

We can, thus, say that the smoothing spline method provides global robustness to noise-like data. The accuracy results on a real video sequence show the improvement of our smoothing spline method over a direct regularization of the segmentation criterion.

\section{CONCLUSION}

In this paper, we address the problem of image and video segmentation by working out a new region-based method using cubic smoothing spline active contours.

Instead of spline interpolation, we have chosen a smoothing spline approximation because we want the method to be more 


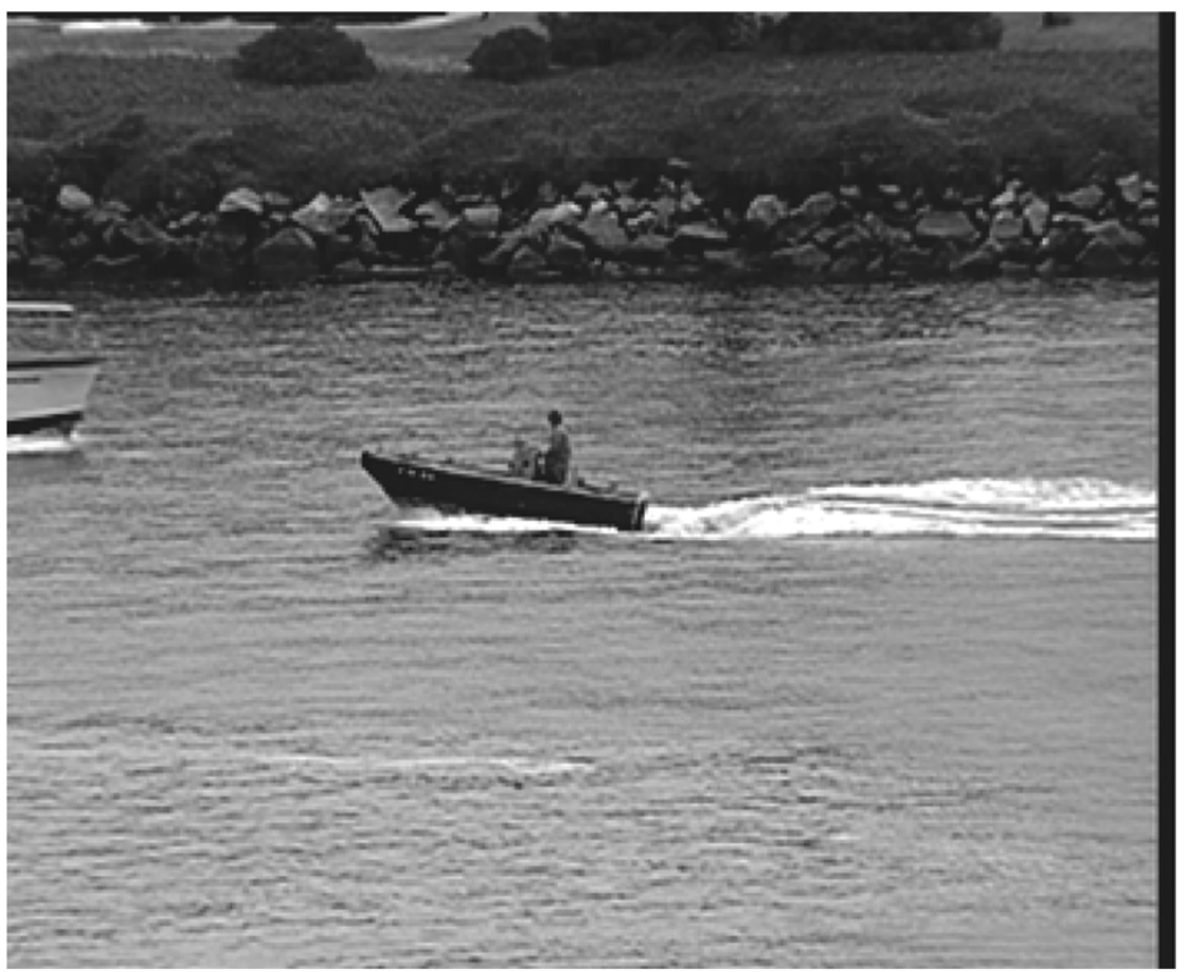

(a)

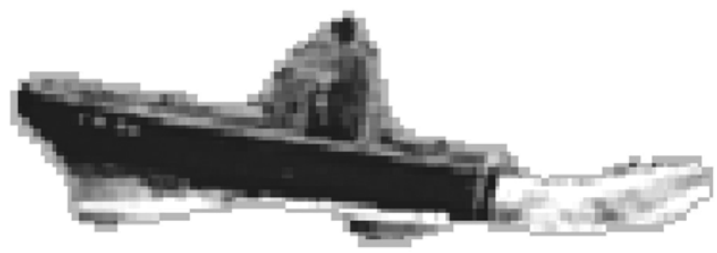

(b)

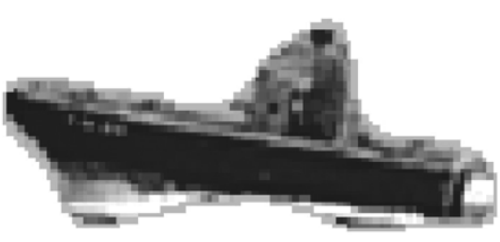

(c)

Fig. 11. Smoothing spline to smooth contours. (a) Initial sequence. (b) Interpolation $\lambda=0$ and $\beta=20$. (c) Approximation $\lambda=0.3$ and $\beta=0$.

robust in the presence of noise. The smoothing spline parameter $\lambda$ provides a tunable tradeoff between interpolation error and contour smoothness. Furthermore, increasing the smoothing spline parameter $\lambda$ improves the sampling uniformity of the contour and avoids the presence of loops. The structure of the contour remains stable to variations of this parameter which is not true with variations of $\beta$, the weight on the length penalty. The robustness of the active contour with respect to $\lambda$ suggested that we could determine a range for standard variations of this parameter. Our experiments provided a range of $[0.1,1]$. As a consequence of the very low computational cost of the B-spline implementation, real-time segmentation is achieved.

\section{APPENDIX I \\ CUBIC SPLINE INTERPOLATION}

\section{A. Recursive Filter Factorization}

Assuming that the data points $P$ are uniformly sampled, the interpolating cubic spline filter $\left(B_{1}^{3}(z)\right)^{-1}$, given in (10), can be factorized into a product of causal and anticausal filters [33]

$$
\left(B_{1}^{3}(z)\right)^{-1}=\frac{6}{z+4+z^{-1}}=\left(\frac{-6 z_{1}}{1-z_{1} z}\right)\left(\frac{1}{1-z_{1} z^{-1}}\right)
$$

where $z_{1}=-2+\sqrt{3}$.
This factorization results in a cascade of first-order causal and anticausal recursive filters. Thus, given the data points $\{P(k)\}_{, k=0, \ldots, N-1}$, the right-hand-side factorization provides the cubic spline interpolating coefficients $\{Q(k)\}, k=0, \ldots, N-1$ through the following recursive algorithm:

$$
\begin{aligned}
& \tilde{Q}(k)=z_{1} \tilde{Q}(k-1)+P(k) \\
& Q(k)=z_{1} Q(k+1)-6 z_{1} \tilde{Q}(k)
\end{aligned}
$$

for all integer $k$, and where $\tilde{Q}(k)$ are intermediate coefficients. We have to specify the initialization for the two recursions.

\section{B. Initialization}

1) Causal Filtering: The first recursion (27) leads to the following relation:

$$
\begin{aligned}
\tilde{Q}(N-k)= & P(N-k)+z_{1} P(N-k-1) \\
& +z_{1}^{2} P(N-k-2)+\cdots \\
& +z_{1}^{N-1} P(N-k-(N-1)) \\
& +z_{1}^{N} \tilde{Q}(-k) .
\end{aligned}
$$




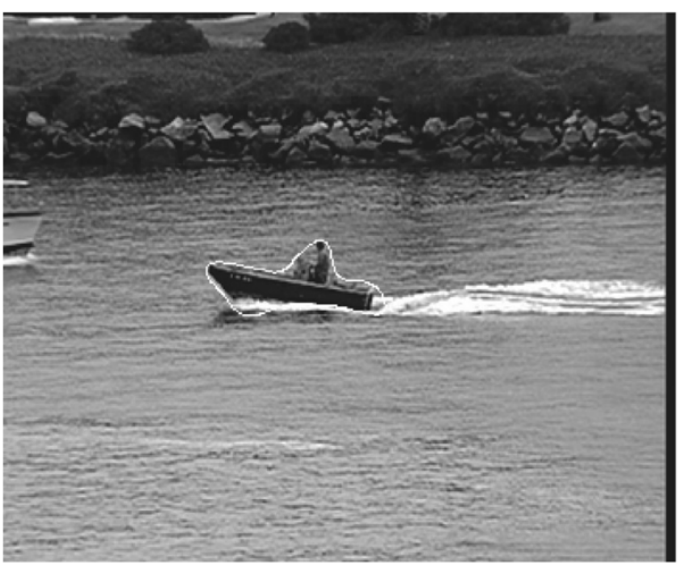

(a)

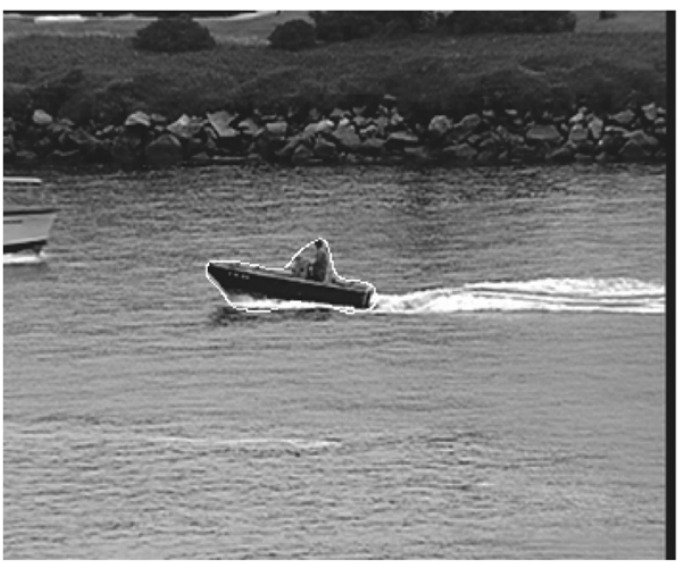

(c)

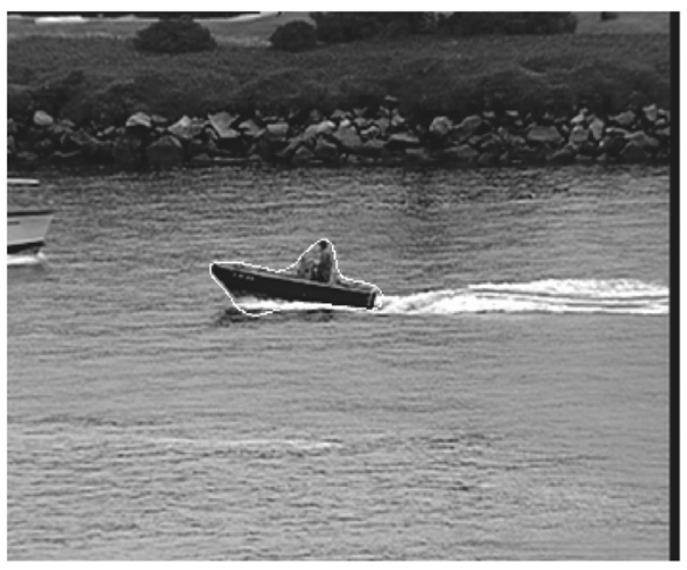

(b)

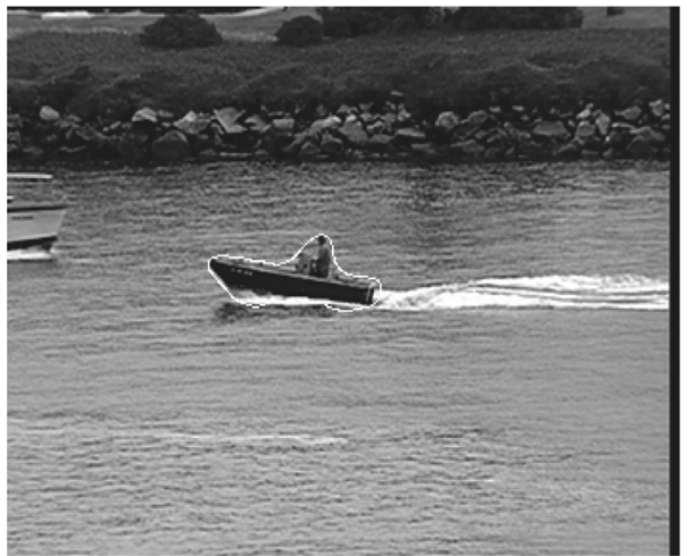

(d)

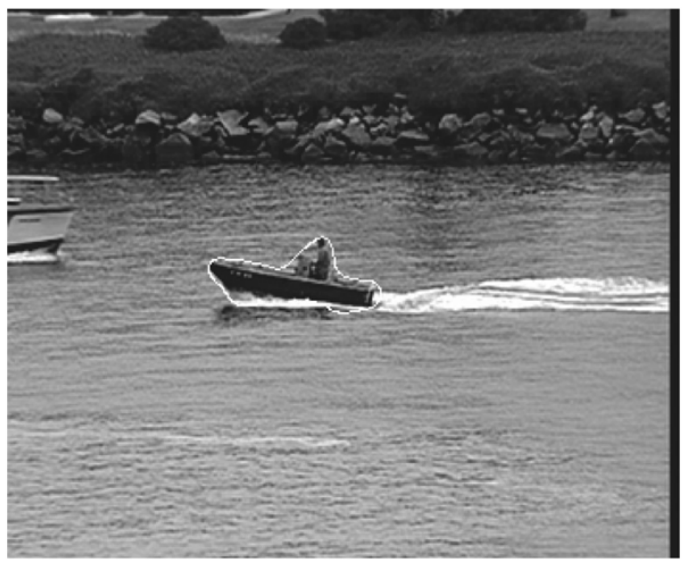

(e)

Fig. 12. Computation time of the segmentation. (a) Frame 1: 1.33 s. (b) Frame 2: 0.12 s. (c) Frame $3: 0.30$ s. (d) Frame $4: 0.42$ s. (e) Frame 5: 0.25 s.

Because the curve is closed, the data points are $N$ periodic, i.e., $P(N-k)=P(-k)$ for all integer $k$. As a result of the filtering operations, the coefficients $\tilde{Q}(k)$ and $Q(k)$ are $N$ periodic, as well.

Letting $k=0$ in (29) and using the $N$ periodicity of $\tilde{Q}(k)$ provides $\tilde{Q}(0)$

$$
\tilde{Q}(0)=\left(\frac{1}{1-z_{1}^{N}}\right) \sum_{l=0}^{N-1} z_{1}^{l} P(N-l) .
$$

The others coefficients $\tilde{Q}(1), \tilde{Q}(2), \ldots, \tilde{Q}(N-1)$ are obtained by applying the induction (27).
2) Anticausal Filtering: We now apply the anticausal filter (28) on the coefficients $\tilde{Q}(k)$ and, in order to trigger the recursion, we need to initialize it by providing the value of $Q(N)=$ $Q(0)$.

Similarly as for the causal case, we obtain the following expression for $Q(0)$ :

$$
Q(0)=Q(N)=-\left(\frac{6 z_{1}}{1-z_{1}^{N}}\right) \sum_{l=0}^{N-1} z_{1}^{l} \tilde{Q}(l) .
$$

Then, the induction (28) provides $Q(N-1), Q(N-2) \ldots Q(1)$. 
We have, thus, specified the appropriate starting values for both causal and anticausal filtering. We cascade these filters with data points $P$ to compute cubic spline coefficients $Q$. The recursive algorithm is stable numerically, fast and easy to implement.

\section{APPENDIX II}

\section{Cubic SMOOTHING SPLine ApPRoximation}

\section{A. Recursive Filter Factorization}

Assuming that the data points $P$ are uniformly sampled, the smoothing cubic spline filter $\left(S_{\lambda}^{3}(z)\right)^{-1}$, given in (15), can be factorized into a product of causal and anticausal filters.

Let us consider the denominator of $\left(S_{\lambda}^{3}(z)\right)^{-1}$

$$
D(z)=z+4+z^{-1}+6 \lambda\left(z^{2}-4 z+6-4 z^{-1}+z^{-2}\right) .
$$

This polynomial can be factorized as

$$
\begin{aligned}
D(z) & =\frac{6 \lambda}{b}\left(1+a z+b z^{2}\right)\left(1+a z^{-1}+b z^{-2}\right) \\
& =\frac{6 \lambda}{b} S_{1}\left(z^{-1}\right) S_{1}(z)
\end{aligned}
$$

where $a$ and $b$ are real numbers. Moreover, because $D(z)$ does not cancel on the unit circle, $S_{1}(z)$ has its roots-whether real or complex-strictly inside the unit circle. This shows that the smoothing spline prefilter can be implemented as a cascade of second-order causal and anticausal recursive filters

$$
S_{\lambda}^{3}(z)=\frac{b}{\lambda} \cdot \frac{1}{1+a z^{-1}+b z^{-2}} \cdot \frac{1}{1+a z+b z^{2}} .
$$

Note that, by defining $x=z-2+z^{-1}$, we can rewrite (32) as $D(z)=6 \lambda x^{2}+x+6$ the roots of which are either real, when $\lambda \leq 1 / 144$, or complex, when $\lambda>1 / 144$. This implies that the roots of $S_{1}(z)$ are either real, when $\lambda \leq 1 / 144$, or complex, when $\lambda>1 / 144$. It is only when $\lambda=1 / 144$ that $S_{1}(z)$ has double roots.

Given the data points $\{P(k)\}, k=0, \ldots, N-1$, the right-hand-side factorization of (34) leads to the cubic smoothing spline coefficients $\{Q(k)\}_{, k=0, \ldots, N-1}$ by the following recursive algorithm:

$$
\begin{aligned}
& \tilde{Q}(k)=-a \tilde{Q}(k-1)-b \tilde{Q}(k-2)+P(k) \\
& Q(k)=-a Q(k+1)-b Q(k+2)+\frac{b}{\lambda} \tilde{Q}(k) .
\end{aligned}
$$

We now have to specify the appropriate initialization for the two recursions.

\section{B. Initialization}

In order to determine the initialization of the recursive filtering algorithm, we need to compute the impulse response $s_{1}(n)$ of the causal filter $S_{1}(z)^{-1}$ in the cascade expression (34).

- When $\lambda=1 / 144, S_{1}(z)=\left(1-z_{0} z^{-1}\right)^{2}$ with $z_{0}=$ $-5+2 \sqrt{6}$ and we immediately have

$$
\frac{1}{S_{1}(z)}=\sum_{n \geq 0}(n+1) z_{0}^{n} z^{-n}
$$

from which we obtain

$$
s_{1}(n)=(n+1) z_{0}^{n} u(n)
$$

where $u(n)$ is the discrete step sequence $u(n)=1$ for $n \geq 0$ and $u(n)=0$ otherwise.

- When $\lambda \neq 1 / 144$, the two roots $z_{0}$ and $z_{1}$ of $S_{1}(z)$ are distinct. We can, thus, decompose $S_{1}(z)^{-1}$ in simple fractions

$$
\frac{1}{S_{1}(z)}=\frac{A}{1-z_{0} z^{-1}}+\frac{B}{1-z_{1} z^{-1}}
$$

where $A=\left(1-z_{1} z_{0}^{-1}\right)^{-1}$ and $B=\left(1-z_{0} z_{1}^{-1}\right)^{-1}$. As a result, the impulse response of $S_{1}(z)^{-1}$ is given by

$$
s_{1}(n)=A z_{0}^{n} u(n)+B z_{1}^{n} u(n) .
$$

Note that the impulse response of the anticausal filter $S_{1}\left(z^{-1}\right)^{-1}$ (needed for the initialization of the anticausal recursion) is given by $s_{1}(-n)$.

1) Causal Recursion: The initialization of (35) requires computing $\tilde{Q}(0)$ and $\tilde{Q}(1)$. Using the impulse response of $S_{1}(z)^{-1}$, we have

$$
\begin{aligned}
& \tilde{Q}(0)=\sum_{n \in \mathbb{Z}} s_{1}(-n) P(n) \\
& \tilde{Q}(1)=\sum_{n \in \mathbb{Z}} s_{1}(1-n) P(n)
\end{aligned}
$$

then, using the $N$ periodicity of $P(n)$ (closed contour)

$$
\begin{aligned}
& \tilde{Q}(0)=\sum_{n_{0}=0}^{N-1} P\left(n_{0}\right) \sum_{n \in \mathbb{Z}} s_{1}\left(-n_{0}+n N\right) \\
& \tilde{Q}(1)=\sum_{n_{0}=0}^{N-1} P\left(n_{0}\right) \sum_{n \in \mathbb{Z}} s_{1}\left(1-n_{0}+n N\right) .
\end{aligned}
$$

We, thus, need to compute an expression of the form $\sum_{n} s_{1}(k+$ $n N)$. For this, we consider the functions

$$
g_{k}(r)=\sum_{n \in \mathbb{Z}} r^{k+n N} u(k+n N) \text { with }|r|<1 .
$$

Since $g_{k}(r)$ obviously satisfies $g_{k}(r)=g_{k+N}(r)$, we can restrict $k$ to $[0, N-1]$ and we find

$$
g_{k}(r)=\sum_{n \geq 0} r^{k+n N}=\frac{r^{k}}{1-r^{N}} .
$$

Thanks to the $N$ periodicity, $k$ has to be replaced by $(k \bmod N)$ in this expression when $k \notin[0, N-1]$. Moreover, by simple differentiation of $g_{k+1}(r)$, we also have that

$$
\begin{aligned}
\sum_{n \in \mathbb{Z}}(k+n N+1) r^{k+n N} u(k+n N) & \\
= & g_{k+1}^{\prime}(r)=\frac{(k+1) r^{k}}{1-r^{N}}+\frac{N r^{N+k}}{\left(1-r^{N}\right)^{2}} .
\end{aligned}
$$

Finally, we find that

- when $\lambda=1 / 144$

$$
\sum_{n \in \mathbb{Z}} s_{1}(k+n N)=g_{(k \bmod N)+1}^{\prime}\left(z_{0}\right) ;
$$




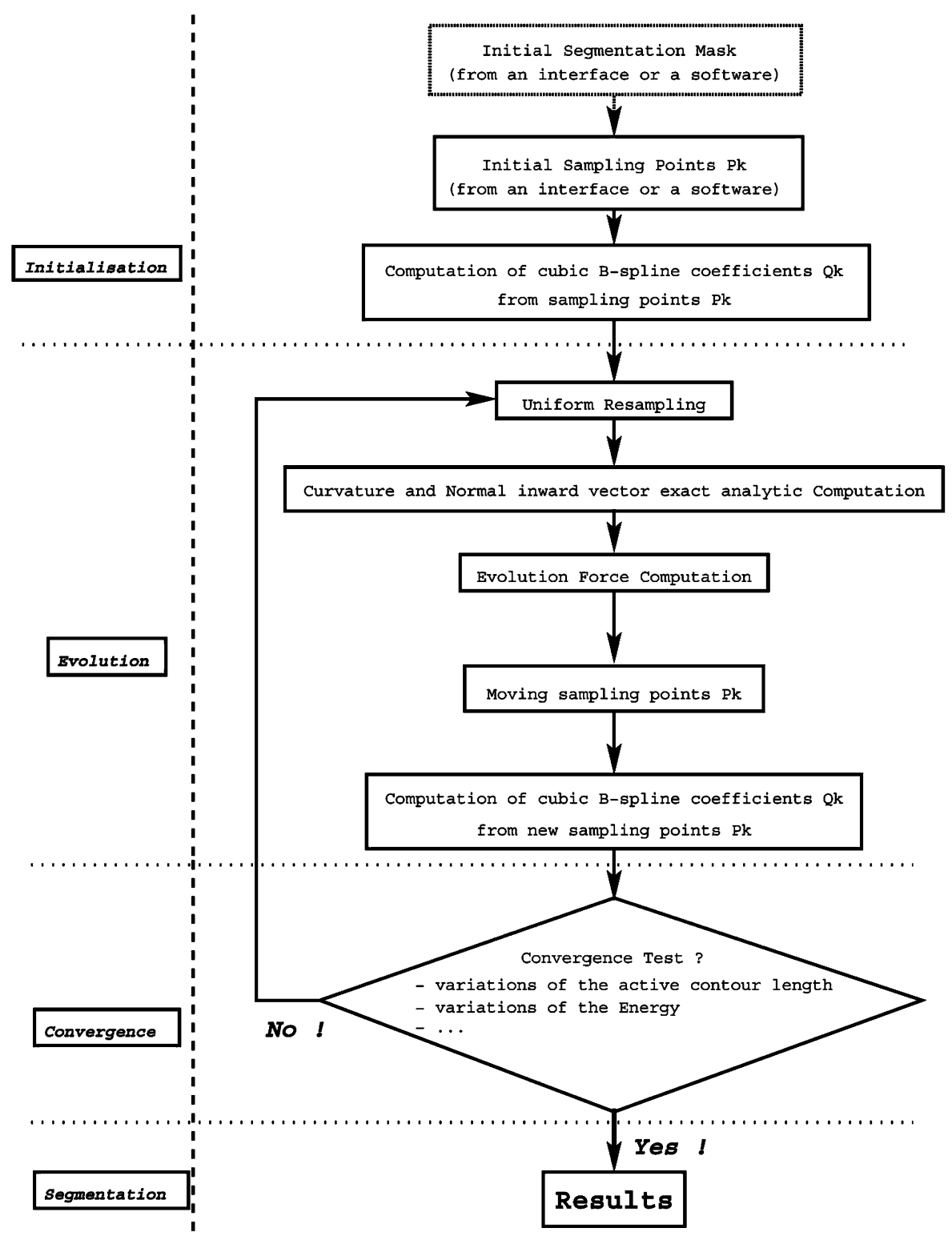

Fig. 13. Flow-chart of our algorithm based on cubic spline interpolation.

- when $\lambda \neq 1 / 144$

$$
\sum_{n \in \mathbb{Z}} s_{1}(k+n N)=A g_{k \bmod N}\left(z_{0}\right)+B g_{k \bmod N}\left(z_{1}\right) .
$$

These expressions can be substituted in (37) to provide the initial conditions to the recursion (35).

2) Anticausal Recursion: The initialization of (36) requires computing $Q(N-1)$ and $Q(N-2)$. Using the impulse response $s_{1}(-n)$ of $S_{1}\left(z^{-1}\right)^{-1}$, we obtain

$$
\begin{aligned}
& Q(N-1)=\frac{b}{\lambda} \sum_{n \in \mathbb{Z}} s_{1}(-(N-1-n)) \tilde{Q}(n) \\
& Q(N-2)=\frac{b}{\lambda} \sum_{n \in \mathbb{Z}} s_{1}(-(N-2-n)) \tilde{Q}(n)
\end{aligned}
$$

then, using the $N$ periodicity of $\tilde{Q}(n)$ (closed contour)

$$
\begin{aligned}
& Q(N-1)=\frac{b}{\lambda} \sum_{n_{0}=0}^{N-1} \tilde{Q}\left(n_{0}\right) \sum_{n \in \mathbb{Z}} s_{1}\left(n_{0}+1+n N\right) \\
& Q(N-2)=\frac{b}{\lambda} \sum_{n_{0}=0}^{N-1} \tilde{Q}\left(n_{0}\right) \sum_{n \in \mathbb{Z}} s_{1}\left(n_{0}+2+n N\right) .
\end{aligned}
$$

By substituting the expressions found in (39) and (40) in these equations, (41) provides the initial conditions to the recursion (36).

To summarize the process described above, given data points $\{P(n)\}_{n=0 \ldots N-1}$, we cascade a causal and an anticausal filter to compute the cubic smoothing spline coefficients $\{Q(n)\}_{n=0 . . . N-1}$. As for interpolating spline, the recursive algorithm for smoothing spline is stable numerically, fast, and easy to implement. 


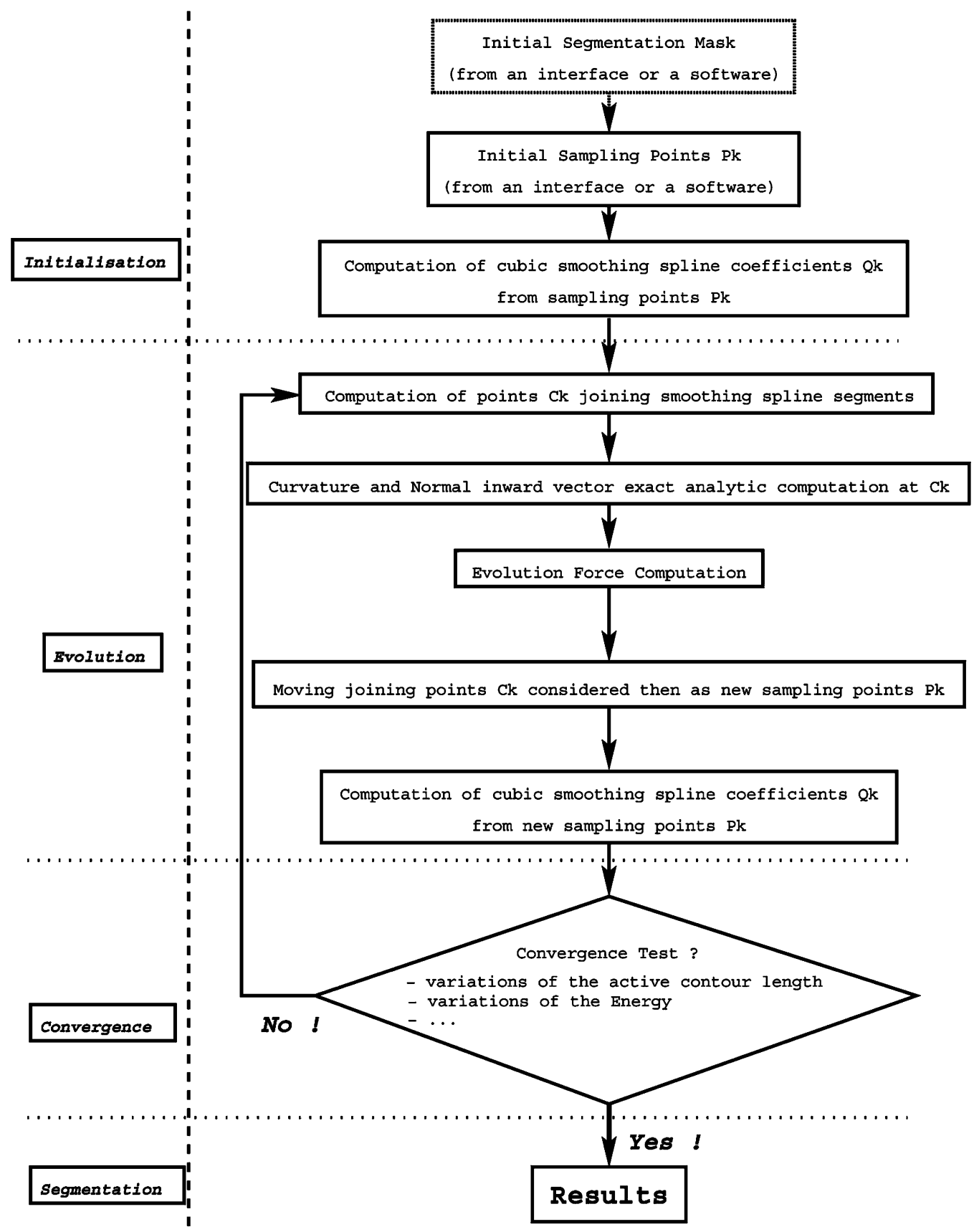

Fig. 14. Flow-chart of our algorithm based on smoothing spline approximation.

APPENDIX III

FLOW-CHARTS OF THE ALGORITHMS

\section{A. Cubic Spline Interpolation}

See Fig. 13.

\section{B. Cubic Smoothing Spline Approximation}

See Fig. 14.

\section{ACKNOWLEDGMENT}

The authors would like to thank A. Herbulot, Ph.D. student at I3S Lab, for her helpful contribution to the section on segmen- tation of homogeneous regions in color video sequences. They would also like to thank DMS for providing osteoprosis images.

\section{REFERENCES}

[1] R. Bartels, J. Beatty, and B. Barsky, An Introduction to Splines for Use in Computer Graphics and Geometric Modeling. San Mateo, CA: Morgan-Kaufmann, 1987.

[2] P. Brigger, J. Hoeg, and M. Unser, "B-spline snakes: A flexible tool for parametric contour detection," IEEE Trans. Image Process., vol. 9, no. 9, pp. 1484-1496, Sep. 2000.

[3] V. Caselles, F. Catte, T. Coll, and F. Dibos, "A geometric model for active contours in image processing," Numer. Math., vol. 66, pp. 1-33, 1993.

[4] V. Caselles, R. Kimmel, and G. Sapiro, "Geodesic active contours," IJCV, vol. 22, no. 1, pp. 61-79, 1997. 
[5] A. Chakraborty, L. Staib, and J. Duncan, "Deformable boundary finding in medical images by integrating gradient and region information," IEEE Trans. Med. Imag., vol. 15, no. 12, pp. 859-870, Dec. 1996.

[6] T. Chan and L. Vese, "Active contours without edges," IEEE Trans. Image Process., vol. 10, no. 2, pp. 266-277, Feb. 2001.

[7] P. Charbonnier, L. Blanc-Féraud, G. Aubert, and M. Barlaud, "Deterministic edge-preserving regularization in computed imaging," IEEE Trans. Image Process., vol. 6, no. 3, pp. 298-311, Mar. 1997.

[8] Y. Chen, H. Tagare, S. Thiruvenkadam, F. Huang, D. Wilson, K. Gopinath, R. Briggs, and E. Geiser, "Using prior shapes in geometric active contours in a variational framework," IJCV, vol. 50, no. 3, pp. 315-328, 2002.

[9] C. Chesnaud, P. Refregier, and V. Boulet, "Statistical region snake-based segmentation adapted to different physical noise models," IEEE Trans. Pattern Anal. Mach. Intell., vol. 21, no. 11, pp. 1145-1156, Nov. 1999.

[10] L. Cohen, E. Bardinet, and N. Ayache, "Surface reconstruction using active contour models," presented at the SPIE Conf. Geometric Methods in Computer Vision, San Diego, CA, 1993.

[11] D. Cremers, C. Schnorr, and J. Weickert, "Diffusion-snakes: Combining statistical shape knowledge and image information in a variational framework," in Proc. 1st IEEE Workshop on Variational and Level Set Methods in Computer Vision, Vancouver, BC, Canada, 2001, pp. 137-144.

[12] E. Debreuve, M. Barlaud, G. Aubert, and J. Darcourt, "Space time segmentation using level set active contours applied to myocardial gated SPECT," IEEE Trans. Med. Imag., vol. 20, no. 7, pp. 643-659, Jul. 2001.

[13] M. Gastaud, M. Barlaud, and G. Aubert, "Combining shape prior and statistical features for active contour segmentation," IEEE Trans. Circuits Syst. Video Technol., to be published.

[14] A. Herbulot, S. Jehan-Besson, M. Barlaud, and G. Aubert, "Shape gradient for image segmentation using information theory," in Proc. Int. Conf. Acoustics, Speech, and Signal Processing, Montreal, QC, Canada, May 2004.

[15] M. Jacob, T. Blu, and M. Unser, "An exact method for computing the area moments of wavelet and spline curves," IEEE Trans. Pattern Anal. Mach. Intell., vol. 23, no. 6, pp. 633-642, Jun. 2001.

[16] _ , "A unifying approach and interface for spline-based snakes ," in Proc. SPIE Int. Symp. Medical Imaging, vol. 4322, San Diego, CA, 2001, pp. 340-347.

[17] S. Jehan-Besson, M. Barlaud, and G. Aubert, "Video object segmentation using Eulerian region-based active contours," presented at the Int. Conf. Computer Vision, Vancouver, BC, Canada, Oct. 2001

[18] _ , "A 3-step algorithm using region-based active contours for video objects detection," EURASIP J. Appl. Signal Process., vol. 2002, no. 6, pp. 572-581, Jun. 2002.

[19] — "DREAM ${ }^{2} \mathrm{~S}$ : Deformable regions driven by an Eulerian accurate minimization method for image and video segmentation, application to face detection in color video sequences," presented at the ECCV, Copenhagen, Denmark, May 2002.

[20] - "DREAM ${ }^{2} \mathrm{~S}$ : Deformable regions driven by an Eulerian accurate minimization method for image and video segmentation," IJCV, vol. 53, no. 1, pp. 45-70, Jun. 2003.

[21] M. Kass, A. Witkin, and D. Terzopoulos, "Snakes: Active contour models," IJCV, vol. 1, pp. 321-332, 1988

[22] J. Kim, J. Fisher III,, A. Yezzi, M. Cetin, and A. Willsky, "Nonparametric methods for image segmentation using information theory and curve evolution," presented at the Int. Conf. Image Processing, Rochester, NY, Sep. 2002.

[23] S. Osher and J. Sethian, "Fronts propagating with curvature-dependent speed: Algorithms based on Hamilton-Jacobi formulation," J. Comput. Phys., vol. 79, pp. 12-49, 1988.

[24] N. Paragios and R. Deriche, "Geodesic active regions and level set methods for supervised texture segmentation," Int. J. Comput. Vis., vol. 46 , no. 3, pp. 223-223, 2002.

[25] H. Pottmann, S. Leopoldseder, and M. Hofer, "Approximation with active B-spline curves and surfaces," in Proc. 10th Pacific Conf. Computer Graphics Applications, Beijing, China, Oct. 2002, pp. 8-24.

[26] F. Precioso and M. Barlaud, "Regular B-spline active contours for fast video segmentation," presented at the Int. Conf. Image Processing, Rochester, NY, Sep. 2002.

[27] — "B-spline active contours with handling of topology changes for fast video segmentation," EURASIP Special Issue: Image Analysis for Multimedia Interactive Services - Part II, vol. 2002, no. 6, pp. 555-560, Jun. 2002.

[28] F. Precioso, M. Barlaud, T. Blu, and M. Unser, "Smoothing B-spline active contour for fast and robust image and video segmentation," presented at the Int. Conf. Image Processing, Barcelona, Spain, Sep. 2003.
[29] C. Reinsch, "Smoothing by spline functions," Numer. Math., vol. 10, pp. $177-183,1967$.

[30] R. Ronfard, "Region-based strategies for active contour models," IJCV vol. 13, no. 2, pp. 229-251, 1994.

[31] I. Schoenberg, "Spline functions and the problem of graduation," Proc. Nat. Acad. Sci., vol. 52, pp. 947-950, 1964.

[32] P. Thévenaz, T. Blu, and M. Unser, "Interpolation revisited," IEEE Trans. Med. Imag., vol. 19, no. 7, pp. 739-758, Jul. 2000.

[33] M. Unser, A. Aldroubi, and M. Eden, "B-spline signal processing: Part I-theory; part II-efficient design and applications," IEEE Trans. Signal Process., vol. 41, no. 2, pp. 821-848, Feb. 1993.

[34] H. Yang, W. Wang, and J. Sun, "Control point adjustment for B-spline curve approximation," Computer Aided-Design, 2004, to be published.

[35] S. Zhu and A. Yuille, "Region competition: Unifying snakes, region growing, and bayes/MDL for multiband image segmentation," in Pattern Anal. Mach. Intell., vol. 18, Sep. 1996, pp. 884-900.

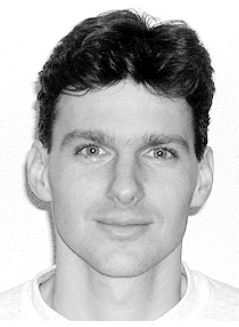

Frederic Precioso was born in 1974. He received the M.S. degree in image processing and computer science from the University of Nice, Sophia Antipolis, France, in 2000. He is currently pursuing the Ph.D. degree at the Laboratory I3S, University of Nice.

$\mathrm{He}$ is currently working on parametric active contour methods for images and videos segmentation. His research interests deal with image and video processing and computer vision.

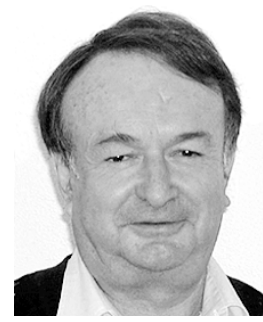

Michel Barlaud received the These d'Etat degree from the University of Paris XII and Agrégation de Physique (ENS Cachan), France.

$\mathrm{He}$ is currently a Professor of image processing at the University of Nice Sophia Antipolis, France, and the Leader of the Image Processing Group CReATIVe of I3S Laboratory. He is a regular reviewer for several journals a member of the technical committees of several scientific conferences. He leads several national research and development projects with French industries and participates in several international academic collaborations (including the University of Maryland, College Park; Stanford University, Stanford, CA; Boston University, Boston, MA; and the Université catholique de Louvain, Louvain, Belgium) and European Research Networks (including COST, Schema, and Similar). He is the author of a large number of publications in the area of image and video processing and Editor of the book Wavelets and Image Communication (New York: Elsevier, 1994). His research topics include image and video coding using scan-based wavelet transforms, inverse problems using half quadratic regularization, and image and video segmentation using region-based active contours and shape gradients.

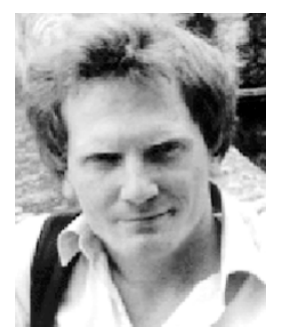

Thierry Blu (M'96) was born in Orléans, France, in 1964. He received the "Diplôme d'ingénieur" from the École Polytechnique, France, in 1986 and from Télécom Paris (ENST), France, in 1988 and the Ph.D. degree in electrical engineering from ENST in 1996 for a study on iterated rational filterbanks, applied to wideband audio coding.

$\mathrm{He}$ is with the Biomedical Imaging Group, Swiss Federal Institute of Technology, Lausanne, Switzerland, on leave from the France Télécom National Center for Telecommunications Studies (CNET), Issy-les-Moulineaux, France. His research interests include (multi)wavelets, multiresolution analysis, multirate filterbanks, approximation and sampling theory, psychoacoustics, optics, and wave propagation.

Dr. Blu is currently an Associate Editor for the IEEE TRANSACTIONS ON IMAGE PROCESSING. 


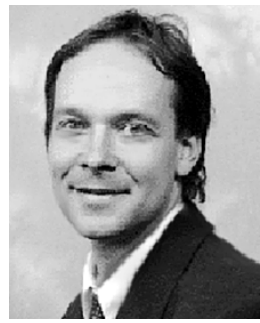

Michael Unser (M'89-SM'94-F'99) received the M.S. (summa cum laude) and Ph.D. degrees in electrical engineering from the Swiss Federal Institute of Technology (EPFL), Lausanne, Switzerland, in 1981 and 1984 , respectively.

From 1985 to 1997, he was with the Biomedical Engineering and Instrumentation Program, National Institutes of Health, Bethesda, MD, where he headed the Image Processing Group. He is now Professor and Director of the Biomedical Imaging Group, EPFL. $\mathrm{He}$ is the Editor-in-Chief of the Wavelet Digest, the electronic newsletter of the wavelet community. He serves as regular chair for SPIE's Conference on Wavelets, which has been held annually since 1993. He has acted as Associate Editor or member of the editorial boards for eight more international journals. His research area is biomedical image processing. He has a strong interest in sampling theories, multiresolution algorithms, wavelets, and the use of splines for image processing, and he is the author of over 100 published journal papers in these areas.

Dr. Unser is the Associate Editor-in-Chief of the IEEE TRANSACTIONS ON MEDICAL IMAGING. He has acted as Associate Editor or member of the editorial boards for the IEEE Signal Processing MAGAZINE, the IEEE TRANSACTIONS ON IMAGe Processing (1992 to 1995), and the IEEE SignAl Processing LETTERS (1994 to 1998). He was general Co-Chair for the first IEEE International Symposium on Biomedical Imaging (ISBI'2002), Washington, DC, July 7-10, 2002. He received the IEEE Signal Processing Society's 1995 Best Paper Award and the IEEE Signal Processing Society's 2000 Magazine Award. In January 1999, he was elected Fellow of the IEEE with the citation "for contributions to the theory and practice of splines in signal processing." 